\title{
STUDY OF THE FUNCTIONAL OUTCOME OF SURGICAL MANAGEMENT OF INTRA-ARTICULAR DISTAL HUMERUS FRACTURE (AO/OTA TYPE C) IN ADULTS
}

\section{Orthopaedics \\ Dr Ravindra Prasad*}

Senior Resident, Department of Orthopaedics, Rajendra Institute of Medical Sciences, Ranchi, Jharkhand. *Corresponding Author

\section{Dr L B Manjhi}

Professor, Department of Orthopaedics, Rajendra Institute of Medical Sciences, Ranchi, Jharkhand.

\section{ABSTRACT}

Introduction: Distal humerus fracture in adults particularly complete articular (AO/OTA Type C) remain some of the most difficult injuries to manage. Complex anatomy of distal humerus combined with multifragmented fracture, sparse soft tissue cover with adjacent neurovascular structures poses great difficulty for treatment. Goal of treatment is to obtain a painless, stable and mobile elbow joint through a systematic approach. Aim: to study the functional outcome of surgical management of complete articular distal humerus fracture in adults. Method: a prospective study comprising of 20 patients treated surgically with open reduction and internal fixation using Orthogonal plating (dorsolateral and medial 3.5mm LCP) through trans olecranon approach. Result: Mean age of patients was 36.6 years (range 19 to 58 years) with male dominance, most fractures were of type 13C1 (AO/OTA). RTA accounts for most common mode of injury, majority of them being left sided. Mean operative time was 130 minutes. Complications included one case of superficial infection, 3 cases of ulnar neuropathy and non-union in 2 cases. Mean range of motion of elbow was 89 degree. Functional outcome assessed using Mayo Elbow Performance Score (MEPS) shown Excellent result in 12 cases, good to fair results in 6 and poor result in 2 cases. Conclusion: Anatomical restoration of joint surface and rigid internal fixation with bicolumnar orthogonal plating allowing early range of motion is the key for obtaining good functional results in complete articular distal humerus fractures. However, outcomes do deteriorate with increasing fracture complexity.

\section{KEYWORDS}

distal humerus fracture, complete articular, bicolumnar plating

\section{INTRODUCTION}

Distal humerus fracture in adults particularly complete articular (AO/OTA Type C) remain some of the most difficult injuries to manage. Complex anatomy of distal humerus combined with multifragmented fracture, sparse soft tissue cover with adjacent neurovascular structures poses great difficulty for treatment. In adult population, ORIF of these fractures using modern fixation principles set by AO-ASIF group which include anatomical articular reduction and rigid internal fixation, is considered the standard of treatment. Nonoperative treatment of these fractures is only justified in elderly patients where poor bone quality and articular comminution so called bag of bones precludes operative management and in young patients who are deemed unfit for surgery.

Goal of treatment should be to obtain a painless, stable and mobile elbow joint through a systematic approach. This requires anatomic reconstruction of the articular surface, restoration of the overall geometry of the distal humerus, and stable fixation of the fractured fragments to allow early and full rehabilitation.

The purpose of this study is to assess the functional outcome of surgical management of displaced complete articular fracture of distal humerus (AO/OTA Type C) in adults by using Orthogonal plating through trans olecranon approach.

\section{MATERIALAND METHODS}

The study was conducted at Department of Orthopaedics, Rajendra Institute of Medical Sciences, Ranchi after getting clearance from the Ethics committee. It included 20 patients who met the inclusion and exclusion criteria.

\section{INCLUSION CRITERIA:}

1. Patients with complete articular fracture of the distal end of the humerus (AO/OTAType C)

2. Patients above the age of 18 years

3. Patients medically fit for surgery

\section{EXCLUSION CRITERIA:}

1. Open fractures of the distal humerus

2. Old fractures of the distal humerus

3. Patients not willing for surgery

4. Patients medically unfit for surgery.

On arrival of patients at casualty or at OPD level, a careful history was elicited from the patient and/or attendants to reveal the mechanism of injury and the severity of trauma. The patients were then assessed clinically to evaluate their general condition and the local injury.
The general condition of the patient and the vital signs were recorded. Methodical examination was done to rule out fractures at other sites. Local examination of injured elbow revealed swelling, deformity and loss of function. Any nerve injury was looked for and noted. Palpation revealed, abnormal mobility and crepitus. Distal vascularity was assessed by radial artery pulsations, capillary filling, pallor and paraesthesia at finger tips.

Radiographic study was done taking anteroposterior and lateral x-ray of the involved elbow. The limb was then immobilized in above elbow POP slab with sling. CT scan with $3 \mathrm{D}$ reconstruction was done in selective cases to improve the identification and visualization of fracture patterns.

The patient was taken for surgery after routine preoperative investigations and after obtaining fitness towards surgery. Most of the patients were operated within 2 weeks of admission.

\section{SURGICAL TECHNIQUE:}

Patients were positioned in lateral decubitus with the arm hanging by the side under brachial block or general anaesthesia. Pneumatic tourniquet was used in all cases.

A longitudinal posterior incision was made over distal arm and elbow curving medially around the olecranon and extending $5 \mathrm{~cm}$ distal to its tip. Skin and subcutaneous tissue are retracted to either side to carefully expose the olecranon and triceps tendon.

The ulnar nerve is isolated and fascia over the flexor carpi ulnaris is longitudinally split to enhance the nerve mobility. Ulnar nerve is then gently retracted from its bed with a moist tape.

The subcutaneous border of proximal ulna exposed and the nonarticular portion of the greater sigmoid notch (the bare area) between the olecranon articular facet and the coronoid articular facet is identified. An apex distal chevron olecranon osteotomy was then made into this bare area using saw and osteotome that is approximately $2 \mathrm{~cm}$ from the tip of the olecranon. The osteotomized olecranon fragment was elevated proximally leaving a margin of the triceps tendon on either side to suture upon completion of the surgery.

The fracture hematoma was cautiously removed. Fragments of the humerus were assembled in three steps. First, reduction and fixation of condyle together followed by fixing the medial or lateral epicondylar ridge to the humeral metaphysis. Lastly, reassembled condyles are fixed to the humeral metaphysis. After reducing the condyles, bone 
holding clamp was used to hold them and provisional fixation was done with Kirschner wires. A 4mm partially threaded CC screw was inserted across the reduced condyles. Then, reduction and provisional fixation of the condyles to metaphysis was done using crossed Kirschner wires. Lastly, $3.5 \mathrm{~mm}$ medial and dorsolateral LCP plates were applied to medial and lateral columns. Plates of different lengths were chosen to minimize the stress riser effect. To enhance the mechanical strength of fixation, the plates were placed as close to 90 degree to each other as possible. The stability of the internal fixation was tested by putting the elbow through a range of motion. The olecranon osteotomy was reduced under direct vision and held with reduction clamp. Periosteum was stripped from the shaft of the ulna distal to the osteotomy site and transverse hole was drilled approximately $3-5 \mathrm{~cm}$ distal to osteotomy site. A No.16 stainless steel malleable wire was passed through thin transverse hole and crossed over the posterior surface of the olecranon in a figure of eight manner and tension band wiring with two Kirschner wires done. At the completion of the fixation, the elbow was again put through a range of motion to test the stability of internal fixation.

Postoperatively, pressure bandage was applied and limb immobilized with Above Elbow POP slab. Active hand range of motion is started immediately and elbow range of motion is started between days 2 and 7 postoperatively.

\section{Follow up:}

Patients were evaluated clinically and radiographically at 2,6 and 12 weeks after surgery. The results were assessed 12 weeks after the procedure. At follow up, a detailed clinical examination was done and patients were assessed subjectively for the symptoms like pain intensity, stability, restriction of joint motion and function. Patients were instructed to carry out physiotherapy in the form of active flexion- extension and pronation-supination without loading. The functional assessment of the patient was done according to Mayo Elbow Performance Score which measures elbow function across four domains: pain (45 points), stability (10 points), range of motion (20 points), and daily functional tasks ( 25 points). Scores are categorized as Excellent (90-100), Good (75-89), Fair (60-74) and Poor (0-59)

\section{RESULT AND DISCUSSION}

Age distribution: The age of the patients in the study ranged from 19 years to 58 years with a mean age of 36.6 years. In our study, fractures were found to be commoner in third and sixth decade.

Sex distribution: Most of the fractures occurred in Males ( 12 out of 20 cases amounting to $60 \%$ ). Other clinical studies also show male predominance of distal humerus fracture.

Mode of Injury: Most common mode of injury was RTA (55\%) followed by simple fall over ground (30\%) and fall from height (15\%).

Side of Injury: Left side was involved in 55\% cases while Right side in $45 \%$ cases.

Type of Fracture: All the distal humerus fracture presenting in our institution were classified as per AO/OTA classification and only Type 13-C (complete articular) were included in the study. Type 13-C1 were most common (55\%), followed by Type 13-C2 (35\%) and Type 13-C3 $(10 \%)$.
C. Complete articular fracture (13-C)

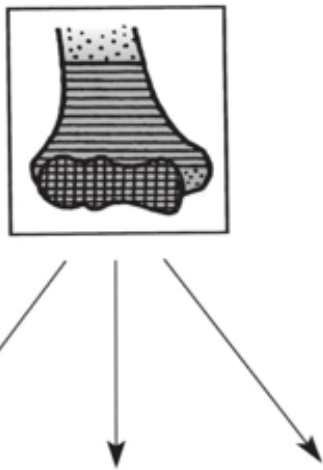

Humerus distal segment, complete articular (13-C)

1. Articular 2. Articular

simple.

metaphyseal

simple (13-C1)

simple,

3. Articular,

physeal multi-

fragmentary

metaphyseal

(13-C2)
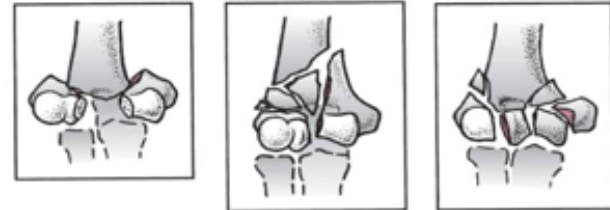

FIGURE 1:AO/OTA CLASSIFICATION DISTAL HUMERUS

Chart 3: Functional Outcome

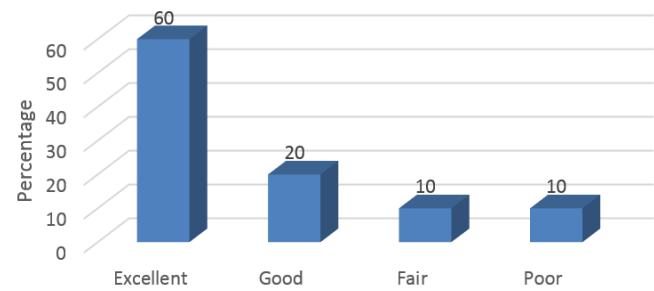

Mayo Elbow Performance Score (MEPS)

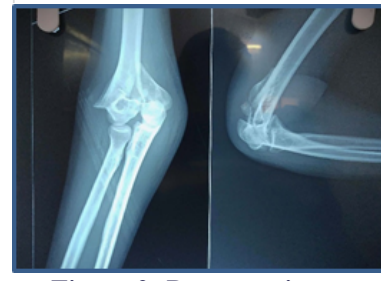

Figure 2: Preoperative Radiograph Distal Humerus

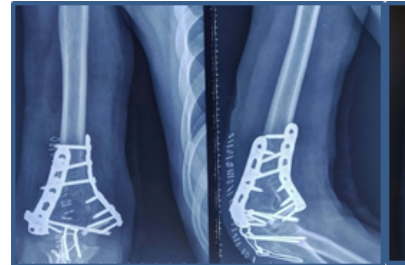

Figure 4: Postoperative Radiograph Day 1

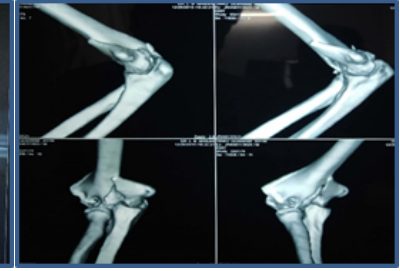

Figure 3: 3D CT Reconstruction

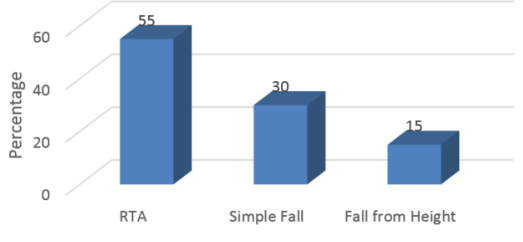

Chart 2: Type of Fracture (AO/OTA)

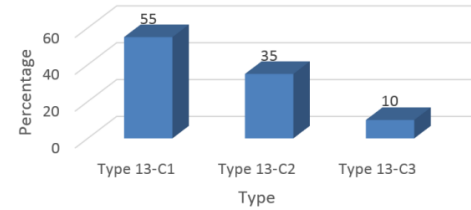

Type 
Ulnar neuropathy: Three cases (15\%) of ulnar neuropathy was seen which resolved spontaneously after conservative treatment. Ulnar nerve injury can occur at the time of fracture, intraoperatively, or postoperatively. Wiggers et al in a retrospective series of 107 patients found that columnar fractures had a greater risk of postoperative ulnar neuropathy than capitellar or trochlear fracture. Chen et al in his retrospective study found a 4 times higher incidence of postoperative ulnar neuropathy in patients who underwent intraoperative ulnar nerve transposition. Hence, these authors recommended against ulnar nerve transposition.

Non-union: Non-union was observed in 2 cases (10\%). In these cases, revision ORIF was done with addition of autologous bone grafting. Risk factors for non-union include inadequate fixation, extensive comminution and severe osteopenia and are reported to occur in approx. $6 \%$ of distal humerus fractures treated by dual plating technique.

Elbow stiffness: Mean range of motion of elbow in our series was 89 degrees ranging from 30 degree to 110 degree. Risk factors for elbow stiffness include head injury, polytrauma, severe soft tissue injury, delayed surgical intervention, prolonged postoperative immobilization and open factures.

Functional Outcome: Mayo Elbow Performance Score (MEPS) used for functional assessment of elbow shown Excellent results in 12 cases $(60 \%)$, Good result in 4 cases $(20 \%)$, Fair results in 2 patients $(10 \%)$ and Poor result in 2 cases $(10 \%)$. Doornberg et al have shown that the rate of good to excellent outcome is durable in the long term. However, there may be some loss of elbow movement in over time in patients sustaining intraarticular fracture. Dubberley et al have reported that outcomes do deteriorate with increasing fracture complexity.

\section{CONCLUSION}

Distal humerus fractures, AO/OTA Type C (complete articular) are complex injuries and ORIF is the gold standard for these injuries as risk of functional impairment is relatively high when these injuries are managed nonoperatively. Surgical fixation of these fractures requires proper preoperative planning, surgical expertise and a systematic approach. Early surgical intervention may lead to decreased complications such as elbow stiffness and heterotopic ossification. Transolecranon approach provides the best visualization of distal humeral articular surface. We conclude that anatomical restoration of joint surface and rigid internal fixation with bicolumnar orthogonal plating allowing early range of motion is the key for obtaining good functional results in complete articular distal humerus fractures. However, outcomes do deteriorate with increasing fracture complexity.

\section{REFERENCES}

1. Pollock JW, Faber KJ, Athwal GS. Distal Humerus Fractures. Orthop Clin North Am. 2008:39(2):187-200.

2. Sanchez-Sotelo J, Torchia ME, O'Driscoll SW Complex distal humeral fractures: Internal fixation with a principle-based parallel-plate technique. J Bone Joint Surg Am. 2007;89(5):961-69.

3. Ilyas AM, Jupiter JB. Treatment of Distal Humerus Fractures. Acta Chir Orthop llyas AM, Jupiter JB. Treatment
Traumatol Cech. 2008;75(1):6-15.

4. Dasheng Tian, Juehua Jing, Jun Qian, Jianming Li. Comparison of two different doubleplate fixation methods with olecranon osteotomy for intercondylar fractures of the distal humeri of young adults. Exp Ther Med. 2013;6(1):147-51

5. Arnander MW, Reeves A, MacLeod IA, Pinto TM, Khaleel A. A biomechanical comparison of plate configuration in distal humerus fractures. J Orthop Trauma. 2008;22(5):332-36.

6. Lee SK, Kim KJ, Park KH, Choy WS. A comparison between orthogonal and parallel plating methods for distal humerus fractures: a prospective randomized trial. Eur J Orthop Surg Traumatol. 2014;24(7):1123-31.

7. Lan X, Zhang LH, Tao S, Zhang Q, Liang XD, Yuan BT, et al. Comparative study of perpendicular versus parallel double plating methods for type $\mathrm{C}$ distal humeral fractures. perpendicular versus parallel double plating

8. Tian D, Jing J, Qian J, Li J. Comparison of two different double-plate fixation methods with olecranon osteotomy for intercondylar fracture of distal humerus of young adults. Exp Ther Med. 2013,

9. Leigey DF, Farrell DJ, Siska PA, Tarkin IS. Bicolumninar 90-90 plating of low energy distal humerus fractures in the elderly patients. Geriatr Orthop Surg Rehabil. 2014;5(3):122-26.

10. Athwal GS, Hoxie SC, Rispoli DM, Steinmann SP. Precontoured parallel plate fixation of AO/OTA type c distal humerus fracture. J Orthop Trauma. 2009;23(8):575-80.

11. Theivendran K, Duggan PJ, Deshmukh SC. Surgical treatment of complex distal humeral fractures: functional outcome after internal fixation using precontoured anatomic plates. J Shoulder Elbow Surg. 2010;19(4):524-32. 